\title{
Artículo de InVestigación \\ Dermatofibrosarcoma Protuberans, análisis clínico e histopatológico de los últimos 14 años en población consultante del Hospital Glínico Universidad de Chile
}

\author{
Fernando Millard ${ }^{1}$,Leonardo Peruilh ${ }^{2}$, Claudia Morales ${ }^{3}$, Fernando Valenzuela ${ }^{2}$
}

\begin{abstract}
RESUMEN
Introducción: El Dermatofibrosarcoma Protuberans (DFSP) es un sarcoma cutáneo infrecuente y poco descrito en la literatura regional. En este trabajo se describió y analizó las características clínicas e histopatológicas de los DFSP tratados en nuestro centro.
\end{abstract}

Métodos: Estudio retrospectivo de todos los casos de DFSP primarios, confirmados con biopsia entre los años 2002 y 2016 en el Servicio de Anatomía Patológica del Hospital Clínico Universidad de Chile (HCUCh).

Resultados: La muestra contó con 18 casos, 67\% de sexo femenino, la edad promedio de diagnóstico fue 45,5 años. El $28 \%$ de los DFSP se localizaron en tronco, $22 \%$ extremidades inferiores, $22 \%$ extremidades superiores, $22 \%$ región inguinogenital y $6 \%$ cervical. Un $22 \%$ de las biopsias fueron enviadas con diagnóstico clínico presuntivo de DFSP. En la histopatología, todos los DFSP estaban compuestos por células fusiformes, distribuidas en patrón estoriforme que infiltraban hasta el tejido adiposo subcutáneo. La inmunohistoquímica se realizó en 7 casos, siendo positivo para CD34 en todas estas muestras y negativo para el factor XIIIa. En el 50\% de los casos se observó compromiso de márgenes quirúrgicos.

Conclusiones: Nuestros resultados fueron similares a publicaciones internacionales. Se observó mayor frecuencia en mujeres y diagnóstico clínico más tardío. Se constató un bajo índice de sospecha clínica de DFSP, confundiéndose con patologías benignas, lo que podría incidir en la frecuencia de márgenes positivos del estudio. Este trabajo constituye uno de los reportes de series de casos más extensos desarrollado a nivel nacional.

Palabras clave: Dermatofibrosarcoma Protuberans; clínica; histopatología e inmunohistoquímica.

Servicio de Dermatología, Hospital Regional de Rancagua. Región del Libertador General Bernardo O’Higgins, Chile. ${ }^{2}$ Departamento de Dermatología, Hospital Clínico Universidad de Chile. Santiago, Chile. ${ }^{3}$ Servicio de Anatomía Patológica, Hospital Clínico Universidad de Chile. Santiago, Chile.

Correspondencia: Fernando Millard San Martín. Correo electrónico: famillard@gmail.com Teléfono: +56 9 82890883. Dirección: Santos Dumont 999, Independencia, Santiago, Chile. Código Postal: 8380456.

\section{SUMMARY}

Background: Dermatofibrosarcoma protuberans (DFSP) is a rare skin sarcoma barely described in regional literature. This paper described and analyzed the clinical and histopathologic features of DFSP treated in our center.

Methods: Retrospective study of all cases of primary DFSP, confirmed with biopsy between 2002 and 2016 in the Department of Pathology of the University of Chile Clinical Hospital (HCUCh).

Results: The sample had 18 cases, $67 \%$ female, the average age of diagnosis was 45.5 years. The $28 \%$ of DFSP were located on the trunk, $22 \%$ lower limbs, $22 \%$ upper limbs, $22 \%$ inguino-genital region and $6 \%$ on the cervical region. A $22 \%$ of the biopsies were sent with presumptive clinical diagnosis of DFSP. On histopathology, all DFSP were composed of spindle-shaped cells distributed in storiform pattern, infiltrating to the subcutaneous adipose tissue. Immunohistochemistry was performed in 7 cases, being positive for CD34 in all of these samples and negative for factor XIIIa. In $50 \%$ of cases surgical margins were positive.

Conclusions: Our results were similar to international publications. Higher frequency was observed in females and a delayed clinical diagnosis. There was a low index of clinical suspicion of DFSP, often confused with benign conditions, which could have influenced the high frequency of positive margins found in the study. This paper is one of the most extensive reports of case series developed nationwide.

Key words: dermatofibrosarcoma protuberans; clinical features; histopathology and immunohistochemistry. 
$\mathrm{E}$ 1 Dermatofibrosarcoma Protuberans (DFSP) es un sarcoma cutáneo infrecuente y poco descrito en la literatura regional. Corresponde a una neoplasia fibrohistiocítica de bajo grado, localmente agresivo, con alta tasa de recidiva local y baja capacidad metastásica ${ }^{1}$. Según estudios internacionales, representa al $1-2 \%$ de todos los sarcomas de tejidos blandos y a menos del $0.1 \%$ de todas las neoplasias malignas ${ }^{1}$. Su incidencia varía de 3 a 6 casos por millón de personas según serie ${ }^{2,5}$, la cual se ha mantenido estable en la última década ${ }^{4}$. El DFSP se presenta a cualquier edad y raza, sin embargo, se produce con mayor frecuencia entre los 20 y 50 años, en personas de raza negra y mujeres ${ }^{1,2,4}$.

En etapas tempranas, este tumor comienza como una placa indurada, asintomática, de color marrón a rojo, que crece lentamente y que presenta una piel atrófica o esclerodermiforme con telangiectasias en su superficie. En etapas tardías, el tumor presenta una mayor velocidad de crecimiento y aparecen múltiples nódulos en la placa, las cuales pueden ulcerarse, sangrar o doler ${ }^{1,6}$. Localmente, estos tumores pueden alcanzar un gran tamaño $(2$ a $5 \mathrm{~cm})$ y pueden adherirse a planos profundo, comprometiendo músculos o huesos ${ }^{1}$.

El DFSP da metástasis en menos del 5\% de los $\operatorname{casos}^{1,9}$, siendo del $1 \%$ para las metástasis regionales y del $4 \%$ para las metástasis a distancia ${ }^{3,6}$. Presenta un comportamiento localmente agresivo y las tasas de recurrencia local varían entre un $0 \%$ a $60 \%$ según el tipo de intervención y márgenes de resección ${ }^{1,10,11}$. El tratamiento ideal para el DFSP localizado, es su extirpación completa con márgenes negativos mediante cirugía convencional, cirugía Micrográfica de Mohs o equivalentes ${ }^{10,12}$. Actualmente se recomienda la cirugía micrográfica de Mohs por su baja tasa de recurrencia $(<1 \%)$, sin embargo, es una técnica compleja y de difícil acceso ${ }^{1,12,13}$. Otra alternativa es la escisión local amplia con márgenes de 2 a $4 \mathrm{~cm}$ y profundización hasta fascia

\section{PUNTOS CLAVE}

En Latinoamérica y en Chile en particular, existen escasos estudios del DSFP.

En este estudio se realizó una descripción de las características clínicas e histopatológicas de los DFSP en un grupo de la población chilena.

Esto podría ayudar caracterizar de mejor forma esta patología, logrando un mayor conocimiento por parte de los profesionales de la salud, permitiendo un diagnóstico precoz y un tratamiento adecuado y efectivo.
El DFSP se localiza principalmen-

te en tronco $(41 \%)$ seguido de extremidades superiores $(21 \%)$, extremidades inferiores $(20 \%)$, cabeza y cuello $(13 \%)$ y genitales $(1 \%)^{1,4,6}$. Otras localizaciones menos frecuentes son en relación a cicatrices o tatuajes y zonas de quemaduras o radiodermatitis ${ }^{1}$.

El diagnóstico clínico de DFSP se confirma mediante una biopsia que evidencia a grupos compactos de células pequeñas, fusiformes con escasa mitosis y dispuestas en patrón "estoriforme" que se extiende desde la dermis hasta el tejido subcutáneo ${ }^{1,6}$. En la grasa subcutánea se observa una infiltración tumoral con patrón de panal de abeja $^{6}$. En la mayoría de los casos, la tinción con Hematoxilina eosina es suficiente para el diagnóstico, sin embargo, es recomendable complementar con estudio inmunohistoquímico. El DFPS habitualmente es positivo para CD34 (84\%-100\% de los casos) y es negativo para factor XIIIa $(90 \% \text { de los casos })^{1,6,8}$. muscular ${ }^{10,12,14}$, presentando una recurrencia del 7\%3,13. La alta tasa de recurrencia se explica por un crecimiento tumoral periférico mediante extensiones digitiformes que clínicamente pasan desapercibidos ${ }^{15,16}$

Debido a lo infrecuente de esta patología y a su presentación clínica inespecífica, el DFSP es habitualmente confundido con patologías benignas, realizándose un diagnóstico tardío y cirugías con mayores secuelas estéticas y funcionales. En Chile existen escasos estudios de series de casos con un tamaño muestral muy pequeño. En el presente trabajo se realizó un extenso reporte de series de casos que permitirá aumentar el conocimiento de la patología en los médicos y eventualmente facilitará un diagnóstico más precoz.

\section{Objetivo}

El objetivo de este trabajo fue describir y analizar las características clínicas, diagnósticos presuntivos y hallazgos histopatológicos de los DFSP del Hospital Clínico Universidad de Chile (HCUCh).

\section{Metodología}

Estudio descriptivo, retrospectivo de todos los casos de DFSP primarios confirmados en el Servicio de Anatomía Patológica del HCUCh entre los años 2002 y 2016. 
Se revisaron todos los informes histopatológicos informados en el servicio y se incorporaron al estudio sólo aquellos con el diagnóstico de DFSP primario, excluyéndose las biopsias de extirpaciones de lesiones residuales o recurrencias. Se procedió a la revisión de fichas clínicas y evaluación de láminas histológicas por un dermatopatólogo del servicio (C.M).

Se estudiaron 18 casos de DFSP, de los cuales se evaluaron variables demográficas (edad y sexo), clínicas (diagnóstico presuntivo previo a confirmación histológica, servicio de derivación, características clínicas y localización anatómica de los DFSP) y variables histopatológicas (diámetro mayor y profundidad tumoral, tipo de proliferación celular, índice mitótico, presencia de pleomorfismo, mitosis atípicas, localización de tumor (de epidermis a músculo), características de la infiltración del tejido subcutáneo y presencia de márgenes quirúrgicos comprometidos). Con respecto a la inmunohistoquímica se evaluó el uso de marcadores CD34, factor XIIIa, desmina, actina y S- 100 .

La tabulación de datos y análisis estadístico se realizó con el programa Microsoft Excel. Para las variables cuantitativas y cualitativas se utilizó estadística descriptiva (números absolutos, promedios, porcentajes y rangos).

\section{Resultados}

Se analizaron 18 casos de DFSP, de los cuales 12 (66.6\%) correspondieron a mujeres y $6(33,4 \%)$ a hombres. La edad promedio en el momento de diagnóstico fue de 45.5 años (rango entre 22-68 años). La edad promedio para mujeres fue de 47.9 años (rango entre 27-68 años) y para los hombres de 40.8 años (rango entre 22-61 años).

Casi la totalidad de los DFSP analizados tenían aspecto clínico de nódulo o tumor $(94,4 \%, \mathrm{n}=17)$ y solo un caso $(5,6 \%)$ se presentó como una placa esclerodermiforme. (Figura 1).

La localización más frecuentemente observada fue en tronco $(\mathrm{n}=5,27,8 \%)$, seguido por las extremidades inferiores $(n=4,22,2 \%)$, extremidades superiores $(n=4$, $22,2 \%)$, región inguino genital $(n=4,22,2 \%)$ y región cervical $(\mathrm{n}=1,5,6 \%)$. (Gráfico 1).
Según género, en mujeres los sitios más afectados fueron tronco, extremidades inferiores y región inguino genital $(\mathrm{n}=3,16,7 \%$ por cada sector), seguido por extremidades superiores $(n=2,11,1 \%)$ y región cervical $(n=1,5,6 \%)$. En el hombre, los sitios más frecuentemente afectados fueron tronco y extremidades superiores $(\mathrm{n}=2,11,1 \%$ para cada sector), seguido por extremidades inferiores y región inguino genital $(\mathrm{n}=1,5,5 \%$ para cada área).

Las biopsias de las lesiones fueron realizadas en el servicio clínico de cirugía en el 66,6\% (n=12) y en dermatología en el 33,4\% (n=6) de los casos. El 22,2\% ( $n=4)$ de las biopsias fueron enviadas con diagnóstico presuntivo de DFSP y el resto con otros diagnósticos como tumor en estudio $(n=5,27,8 \%)$, lipomas $(n=2,11,1 \%)$, queloides $(\mathrm{n}=2,11,1 \%)$, quiste epidérmico $(\mathrm{n}=2,11,1 \%)$, pilomatrixoma $(\mathrm{n}=1,5,6 \%)$, adenopatía $(\mathrm{n}=1,5,6 \%)$ y morfea (n=1, 5,5\%). (Gráfico 2).

En relación a las variables histopatológicas, el diámetro mayor y profundidad tumoral promedio fue $26.8 \mathrm{~mm}$ (rango entre 10-60 mm) y $15 \mathrm{~mm}$ (rango entre 6-30 mm), respectivamente. Todos los DFSP estaban compuestos por una proliferación de células fusiformes, distribuidas en patrón estoriforme y con un índice mitótico menor a 5 en 10 campos de mayor aumento. (Figura 2). En ningún caso se observó pleomorfismo y necrosis tumoral. En 13 casos (72,3\%), la proliferación tumoral respetaba dermis papilar. Todos los tumores comprometían tejido subcutáneo, sin invasión a la fascia muscular. La infiltración hacia el tejido adiposo fue a través de los lobulillos en el 100\% de los casos, adoptando un patrón en panal de abeja. En 5 casos (27,8\%), este patrón se acompañó de un patrón en multicapas. Además, se observó compromiso concomitante de los septos en un 38,9\% $(n=7)$ de las biopsias. (Tabla 1).

En el 50\% (n=9) de los casos se observó compromiso de los márgenes quirúrgicos, ya sea laterales y o profundos.

El diagnóstico de DFSP se realizó con tinción convencional de Hematoxilina y eosina y se complementó con inmunohistoquímica en 7 casos, correspondiendo al 38,9\% de las muestras. Se observó positividad de CD34 en todos los casos y de vimentina en 2 casos. (Figura 2). El estudio de factor XIIIa, S-100, actina y desmina se realizó en 4 muestras y fueron negativas. 


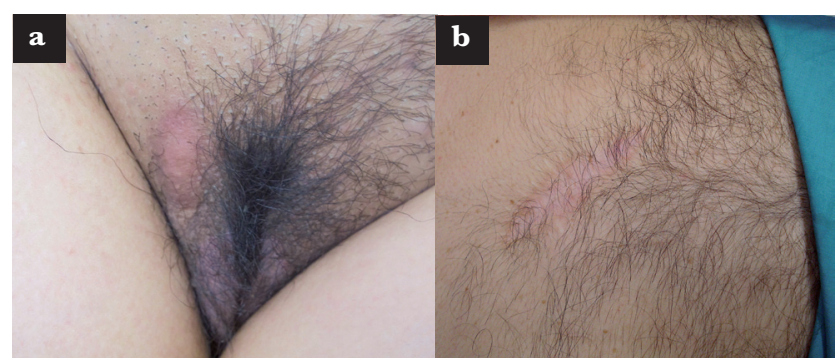

Figura 1

a. Nódulo rosado bien delimitado localizado en pubis.

b. Placa atrófica eritematosa localizado en tórax.

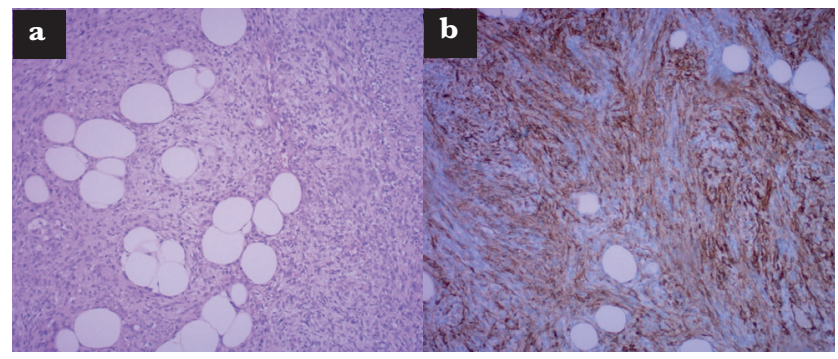

\section{Figura 2}

a. Tinción con Hematoxilina-eosina, 100x. Proliferación de células fusiforme distribuidas en patrón estoriforme, que rodean a las células adiposas.

b. Inmunohistoquímica, tinción con CD34. Tinción positiva en citoplasma de células fusiformes.

Tabla 1. Distribución de hallazgos histológicos.

\begin{tabular}{l|c}
\hline \multicolumn{1}{c|}{ Hallazgos en epidermis } & Total(\%) \\
\hline $\begin{array}{l}\text { Hiperplasia Epidérmica } \\
\text { Respeta dermis papilar o zona de Grenz }\end{array}$ & $14(77,8 \%)$ \\
\hline \multicolumn{1}{c}{ Hallazgos en dermis } & $13(72,3 \%)$ \\
\hline Células fusiforme & $18(100 \%)$ \\
Patrón estoriforme & $18(100 \%)$ \\
Índice mitótico en 10 campos de mayor aumento & \\
\multicolumn{1}{c|}{ Menor a 5 } & $18(100 \%)$ \\
\hline \multicolumn{1}{c}{ Hallazgos a 5} & 0 \\
\hline Infiltración tejido subcutáneo (TSC) & $18(100 \%)$ \\
Infiltración TSC por lobulillos & $18(100 \%)$ \\
Patrón panel de abeja & $18(100 \%)$ \\
Infiltración TSC por septos & $7(38,9 \%)$ \\
Patrón multicapas & $5(27,8 \%)$ \\
\hline
\end{tabular}

\section{Abreviaturas:}

DFSP: Dermatofibrosarcoma Protuberans

HCUCh: Hospital Clínico Universidad de Chile.

\section{Gráfico 1}

Distribución de DFSP según localización.

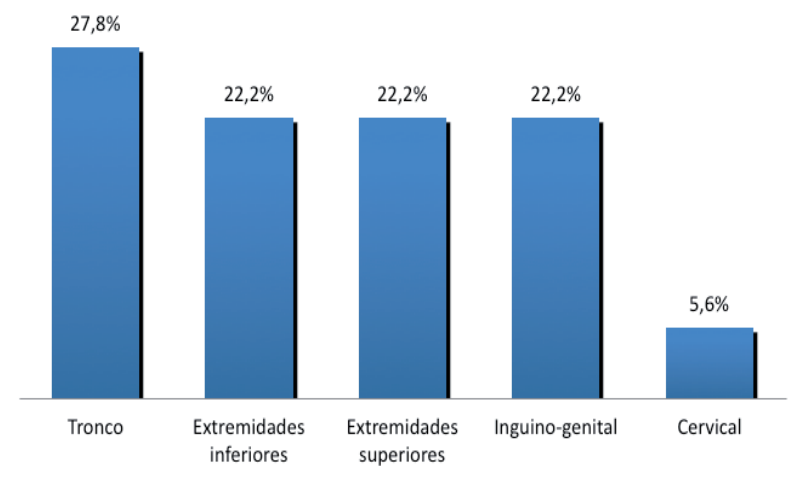

Gráfico 2

Distribución según diagnóstico presuntivo.

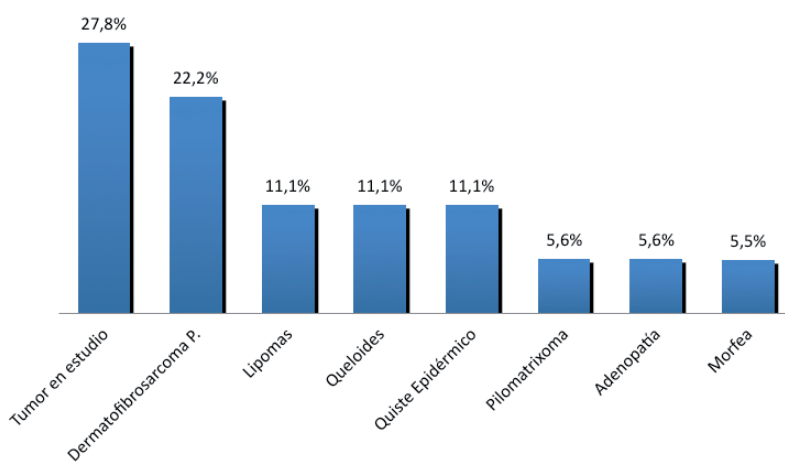

\section{Discusión}

En este trabajo se describió las características demográficas, clínicas e histopatológica de los DFSP primarios en pacientes consultantes del HCUCh. Se observó una mayor frecuencia de DFSP en mujeres (67\%), lo cual coinciden con otras series descritas. Los principales servicios clínicos en donde se obtuvieron las muestras fueron cirugía $(66.6 \%)$ y dermatología $(33.4 \%)$. En relación a la edad promedio del diagnóstico e índice de sospecha clínica del DFSP, en nuestra muestra se observó un diagnóstico clínico más tardío (45,5 años) y se constató un bajo índice de sospecha clínica $(22,2 \%)$ de los DFSP, confundiéndose con patologías benignas como lipomas, quiste epidérmico, queloides, entre otros. Esto podría 
ser explicado por las características clínicas inespecíficas, crecimiento lento y comportamiento indolente de los DFSP ${ }^{1,3,6}$.

Los DFSP se presentaron principalmente como un nódulo o tumor, excepto en un caso, en donde se presentó como una placa tipo morfea like. Con respecto a la localización, la mayor frecuencia observada en ambos sexo fue en tronco con un $27,8 \%$, seguido de un $22,2 \%$ en extremidades inferiores, $22,2 \%$ en extremidades superiores, $22,2 \%$ en región inguino escrotal y $5,6 \%$ en región cervical, resultados que coinciden con los reportes internacionales ${ }^{1,4,6}$.

En relación a la histología, se observó que todos los DFSP estaban compuestos por una proliferación de células fusiformes en patrón estoriforme y sin signos de agresividad histológica (sin mitosis atípica, pleomorfismo o necrosis tumoral). En la mayoría de las muestras (72,3\%), la proliferación tumoral respetaba dermis y en todos los casos comprometía el tejido adiposo subcutáneo a través de los lobulillos. Sin embargo, a diferencia de lo reportado en la literatura internacional, se observó un menor diámetro tumoral y compromiso septal $(38,9 \%)$ del tejido adiposo subcutáneo ${ }^{1}$, posiblemente explicado por la extirpación incompleta del tumor. En el 50\% de los casos se observó compromiso de los márgenes quirúrgicos, similar a lo reportado en algunos estudios ${ }^{17}$. Esto se explica por un bajo índice de sospecha y por el crecimiento irregular del tumor, invadiendo al tejido subcutáneo con extensiones digitiformes que clínicamente pueden pasar desapercibidos ${ }^{15,16}$.

En la mayoría de los casos, el diagnóstico de DFSP se puede realizar con estudio histopatológico convencional con Hematoxilina eosina, sin embargo, es recomendable solicitar en todos los casos estudio inmunohistoquímico con la finalidad de distinguirlo de otras neoplasias mesenquimales y de realizar el mejor tratamiento.

En nuestro estudio, se complementó con estudio inmunohistoquímico en el 38,9\% de las muestras, siendo positivo en todos los casos para CD34 y negativo factor XIIIa, desmina, actina y S-100, resultados concordantes con la literatura internaciona ${ }^{11,6,8}$.

\section{Conclusión}

El DFSP es un sarcoma cutáneo infrecuente y poco descrito en la literatura regional. Es una neoplasia fibro- histiocítica de bajo grado, localmente agresivo, con alta tasa de recidiva local y baja capacidad metastásica. En este estudio se observó una mayor frecuencia en mujeres y diagnóstico clínico más tardío. Además se constató un bajo índice de sospecha clínica de DFSP, confundiéndose con patologías benignas, lo que podría incidir en la frecuencia de márgenes positivos del estudio. La extirpación con márgenes amplios o cirugía micrográfica de Mohs serían los tratamientos de elección.

Este trabajo correspondería a uno de los reportes de series de casos más extensos desarrollado a nivel nacional y esperamos que contribuya en el mejoramiento de las características clínicas e histopatológicas de los DFSP y en aumentar el conocimiento de esta enfermedad por parte de los profesionales de salud.

\section{REFERENCIAS}

1. Llombart B, Serra-Guillen C, Monteagudo C, J.A. Guerrero J, San Martin O. Dermatofibrosarcoma Protuberans: a comprehensive review and update on diagnosis and management. Semin Diagn Pathol 2013;30(1):13-28

2. Criscione V, Weinstock M. Descriptive epidemiology of dermatofibro sarcomaprotuberans in the United States, 1973 to 2002. J Am Acad Dermatol 2007;56(6):968-973

3. Monnier D, Vidal C, Martin L, Danzon A, Pelletier F, Puzenat E, et al. Dermatofibrosarcoma protuberans: a population-based cáncer registry descriptive study of 66 consecutive cases diagnosed between 1982 and 2002. J Eur Acad Dermatol Venereol 2006; 20(10):12371242

4. Kreicher K, Kurlander D, Gittleman H, Barnholtz-Sloan J, Bordeaux J. Dermatofibrosarcoma Protuberans in the United States. Dermatol Surg 2016;42(1);2431

5. Rouhani P, Fletcher, Devesa S, Toro J. Cutaneous soft tissue sarcoma incidence patterns in the U.S.: an analysis of 12,114 cases. Cancer. 2008;113(3):616-627

6. Angouridakis N, Kafas P, Jerjes W, Triaridinis S, Upile T, Karkavelas G, et al. Dermatofibrosarcoma protuberans with fibrosarcomatous transformation of the head and neck. Head Neck Oncol 2011;3:1-7

7. Abenoza P, Lillemoe T. CD34 and factor XIIIa in the differential diagnosis of dermatofibroma and dermatofibrosarcoma Protuberans. Am J Dermatopathol. 1993;15:429-434

8. Aiba S, Tabata N, Ishii H,Ootani H, Tagami H. Dermatofibrosarcoma Protuberans is a unique fibrohistiocytic tumour expressing CD34. Br J Dermatol 1992;127:79-84

9. Arican O, Bakaris S, Bulbuloglu E, Ezberci F. Myoid differentiation and EMA expression in fibrosarcomatous dermatofibrosarcoma Protuberans. Acta Dermatovenerol Alp Pannonica Adriat 2006; 15(1):39-44

10. Miller J, Alam M, Andersen J, Berg D, Bichakjian C, Bowen G, et al. Dermatofibrosarcoma Protuberans. J Natl Compr Canc Netw 2012;10(3):312-318 
11. Loss L, Zeitouni N. Management of scalp dermatofibrosarcoma Protuberans. Dermatol Surg 2005;31(11);1428-1433

12. Paradisi A, Abeni D, Rusciani A,Cigna E, Wolter M, Scuderi N, et al. Dermatofibrosarcoma protuberans: wide local excision vs. Mohs micrographic surgery. Cancer Treat Rev 2008;34(8):728-736

13. Loghdey M, Varma S, Rajpara S, Al-Rawi H, Perks G, Perkins W. Mohs micrographic surgery for dermatofibro sarcomaprotuberans (DFSP): a single-centre series of 76 patients treated by frozen-section Mohs micrographic surgery with a review of the literatura. J Plast Reconstr Aesthet Surg 2014;67(10):1315-1321

14. Kim B, Kim H, Jin U, Minn K, Chang H. Wide Local Excision for Dermatofibrosarcoma Protuberans: A Single-Center Series of 90 Patients. Biomed Res Int 2015;2015:9 pages.
15. Haycox C, Odland P, Olbricht S, Casey B. Dermatofibrosarcoma protuberans (DFSP): growth characteristics based on tumor modeling and a review of cases treated with Mohs micrographicsurgery. Ann Plast Surg. 1997;38(3):246-251

16. Ratner D, Thomas C, Johnson T, Sondak V, Hamilton T, Nelson B, et al. Mohs micrographic surgery for the treatment of Dermatofibrosarcoma protuberans. Results of a multiinstitutional series with ananalysis of the extent of microscopic spread. J Am Acad Dermatol 1997;37:600-613

17. 17. Goldberg C, Hoang D, McRae M, Chung C, Leffell D, Narayan D. A strategy for the successful management of dermatofibrosarcoma Protuberans. Ann PlastSurg 2015;74(1):80-84 